\title{
REPRODUÇÃO DE PEIXES (OSTEICHTHYES) EM AFLUENTES DO RESERVATÓRIO DE VOLTA GRANDE, RIO GRANDE, SUDESTE DO BRASIL
}

\author{
Francisco Manoel de Souza Braga ${ }^{1}$
}

\begin{abstract}
FISHES REPRODUCTION IN THE VOLTA GRANDE DAM TRIBUTARIES, GRANDE RIVER, SOUTHEASTERN BRAZIL. The most abundant species caught in the tributaries of the Volta Grande dam, Buriti, Divisa and Água Comprida streams were Plagioscion squamosissimus (Heckel, 1840), Pimelodus maculatus Lacépède 1803, Myleus tiete (Eigenmann \& Norris, 1900), Astyanax bimaculatus (Linnaeus, 1758) and A. fasciatus (Cuvier, 1819). Plagioscion squamosissimus and Pimelodus maculatus do not have reproduction activity in the area, they just use the creeks to food and growth. Myleus tiete and Astyanax bimaculatus use the area to reproduction whereas $A$. fasciatus uses it to reproduction, food and growth.
\end{abstract}

KEYWORDS. Reproduction, Volta Grande dam, reservoir.

\section{INTRODUÇÃO}

O reservatório de Volta Grande localiza-se no rio Grande, entre os estados de Minas Gerais e São Paulo $\left(19^{\circ} 57^{\prime}, 20^{\circ} 10^{\prime} \mathrm{S}\right.$ e $\left.48^{\circ} 25^{\prime}, 47^{\circ} 35^{\prime} \mathrm{W}\right)$, tendo uma área inundada de aproximadamente $205 \mathrm{~km}^{2}$, um perímetro de $80 \mathrm{~km}$ e volume de $23 \times 10^{9}$ $\mathrm{m}^{3}$; a sua formação data de 1973 (Rolla et al., 1990). Juntamente com outros 10 reservatórios forma um sistema em cascata no rio Grande, que vai de 900 (reservatório de Camargos) até 300 metros acima do nível do mar (reservatório de Água Vermelha). O reservatório de Volta Grande está situado a aproximadamente 500 metros acima do nível do mar, tendo a montante o reservatório de Igarapava e a jusante o reservatório de Porto Colômbia (SAntos \& Formagio, 2000).

Atualmente, o setor elétrico brasileiro tem causado a submersão de alguns milhares de quilômetros quadrados em diversos pontos do território nacional. No rio Tietê, São Paulo, a área inundada é de $1166 \mathrm{~km}^{2}$ (CESP, 1993a) e as onze hidrelétricas em cascata no rio Grande, juntas, perfazem $3511 \mathrm{~km}^{2}$ de área submersa (SANTOS \& Formagio, 2000). As áreas inundadas deixam de produzir elementos agropecuários em um curto espaço de tempo; submergem matas ou outros ecossistemas, inclusive em alguns casos, áreas urbanas; provocam distúrbios ambientais dentro do próprio ecossistema hídrico, interrompendo ciclos reprodutivos de muitas espécies de peixes reofílicos e alterando áreas de desova e de desenvolvimento de larvas. A introdução de espécies exóticas tem causado problemas de super populações, como é o caso da corvina, Plagioscion squamosissimus (Heckel, 1840), que após a sua introdução no nordeste foi trazida para São Paulo e solta no rio Pardo no início dos anos 70. Atualmente é uma espécie abundante em quase toda bacia do Paraná, estando ausente somente em alguns reservatórios do rio Paranapanema, rio Paraíba do Sul e rio Ribeira de Iguape (CesP, 1993b).

Devido aos sucessivos barramentos de rios, as espécies tendem a se adequar às novas situações ecológicas, para poder realizar satisfatoriamente o ciclo reprodutivo

1. Instituto de Biociências, Departamento de Zoologia, Centro de Aqüicultura, Universidade Estadual Paulista, Av. 24 A, 1515, C.P. 199, 13506-900 Rio Claro, SP, Brasil. (fmsbraga@rc.unesp.br) 
(Suzuki \& Agostinho, 1997). Na represa do Lobo, Leporinus friderici (Bloch 1794), espécie tipicamente reofílica (Godoy, 1975), desenvolve todo o ciclo de vida nesse ambiente lêntico (BARBIERI \& GARAVELlo, 1981). Na represa de Barra Bonita, rio Piracicaba, GenNARI-FiLho \& BRAGA (1996) encontraram baixa freqüência de indivíduos maduros de Astyanax schubarti Britski, 1964 em relação à de Astyanax bimaculatus (Linnaeus, 1758). Como A. schubarti tem desova total, a baixa freqüência de indivíduos maduros na represa foi associada a uma possível migração reprodutiva nos tributários. Portanto, quando um ambiente torna-se alterado ou é inapropriado para o desenvolvimento completo do ciclo de vida de uma espécie, esta deve-se adaptar a novas condições para poder ocupar o espaço ou será eliminada desse ecossistema. No rio Grande, que possui sucessivas barragens, diversas espécies de peixes migradores têm utilizado alguns afluentes para a reprodução e por isso esses afluentes devem ser preservados para garantir o processo reprodutivo (SANTOS \& Formagio, 2000).

Alguns afluentes do reservatório de Volta Grande foram estudados, com o objetivo de constatar a ocorrência ou não da atividade reprodutiva e mostrar a importância deles para a manutenção de espécies em um ambiente alterado.

\section{MATERIAL E MÉTODOS}

Nos meses de abril, junho, setembro e dezembro de 1997 e fevereiro de 1998 foram realizadas coletas em três córregos tributários do reservatório de Volta Grande: córrego do Buriti, da Divisa e da Água Comprida (fig. 1). A amostragem foi feita uma vez por período de coleta, utilizando-se cinco conjuntos de redes de emalhar com 150 metros cada um, por 1,5 metro de altura e tendo cada conjunto as seguintes malhagens: 2,3,4,5 e 6 centímetros entre nós adjacentes.

Após as despescas, os peixes foram identificados por espécie e obtido de cada exemplar os seguintes dados: a) comprimento total em centímetros; b) peso total em gramas; c) sexo e estádio de maturidade, considerando-se os estádios imaturo (A), em maturação ou repouso (B), maduro (C) e esgotado (D); d) grau de repleção do estômago, considerando-se os graus sem alimento (1), com alimento (2) e repleto (3); e) grau de gordura acumulada na cavidade visceral, considerando-se os graus sem gordura (1), com gordura (2) e repleto (3). As fêmeas maduras (estádio C) tinham seus ovários pesados até centésimos de grama para posterior estimativa da relação gonadossomática (RGS) dada por: peso do par de ovários/peso total do peixe.100 (VAZzoLER, 1996).

A diversidade das espécies entre locais de coleta foi analisada utilizando-se o índice de diversidade de Shannon estimado por: $H^{\prime}=-\Sigma p_{i} \log p_{i}$ e a variância dada por $S^{2}=\left[\left(\sum \mathrm{f}_{\mathrm{i}} \log ^{2} \mathrm{f}_{\mathrm{i}}\right)-\left(\sum \mathrm{f}_{\mathrm{i}} \cdot \log \mathrm{f}_{\mathrm{i}}\right)^{2} / \mathrm{n}\right] / \mathrm{n}^{2}$. A

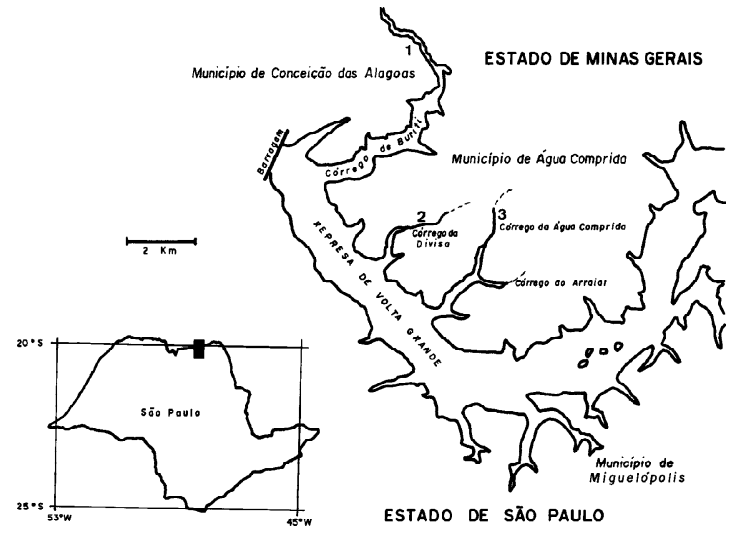

Fig. 1. Localização da represa da Unidade Hidrelétrica de Volta Grande, rio Grande, (MG-SP), com indicação dos locais de coleta $(1,2 \mathrm{e} 3)$. 
equitabilidade, que é o índice que mostra como as categorias específicas estão constituídas por número de indivíduos é dada por $\mathrm{E}=\mathrm{H}^{\prime} / \mathrm{lnS}$, sendo $\mathrm{S}$ o número de espécies por local (MAGURRAm, 1991).

Para as espécies dominantes nos córregos foi avaliada a intensidade reprodutiva por espécie, com o objetivo de verificar se ocorreu atividade reprodutiva e com qual intensidade (VAZZOLER, 1996). Esse método envolve uma sucessão de etapas: a) estimativa da frequiência dos estádios de maturidade durante todo o período para cada espécie dominante; b) estimativa da freqüência de fêmeas maduras (estádio de maturidade C) por período de coleta, em relação aos demais estádios; c) estimativa do valor da relação gonadossomática (RGS) médio para as fêmeas maduras, por período; d) estimativa da porcentagem do valor médio da RGS por período em relação ao valor máximo da RGS no período todo; e) estimativa da categoria reprodutiva em função da frequiência de fêmeas maduras e da porcentagem da RGS máxima, por período, considerando: desova massiva (DM), quando a porcentagem de fêmeas maduras (C) é alta e a porcentagem da RGS máxima é elevada; desova ocasional (DO), quando a porcentagem de fêmeas maduras (C) é baixa e a porcentagem da RGS máxima é elevada; maturação incipiente (MI), quando a porcentagem de fêmeas maduras (C) é alta e a porcentagem da RGS máxima é baixa; sem atividade reprodutiva (SAR), quando a porcentagem de fêmeas maduras (C) e a porcentagem da RGS máxima são baixas; f) estimativa do índice de intensidade reprodutiva, dado por: $I R=\Sigma\left(\mathrm{P} . \mathrm{N}_{\mathrm{sp}}\right) / \Sigma \mathrm{N}_{\mathrm{sp}}$, sendo: $\mathrm{N}_{\mathrm{sp}}=$ número de estratos (espécies) por categoria; $\mathrm{P}=4$ para desova massiva; $\mathrm{P}=2$ para desova ocasional; $\mathrm{P}=1$ para maturação incipiente; $\mathrm{P}=0$ para sem atividade reprodutiva; g) estimativa do comprimento médio da primeira maturação gonadal $\left(\mathrm{L}_{50}\right)$ e da freqüência de jovens e de adultos, por espécie dominante; $h$ ) estimativa da utilização do ecossistema pelas espécies dominantes, considerando-se: área de reprodução coletiva, quando a IR for alta e a porcentagem de jovens for menor que a porcentagem de fêmeas adultas e área de alimentação e crescimento quando a IR for baixa e a porcentagem de jovens for maior que a porcentagem de fêmeas adultas.

Dados ambientais como temperatura do ar e pluviométricos foram obtidos durante o período de coleta, junto ao posto meteorológico da Unidade Hidrelétrica de Volta Grande.

\section{RESULTADOS}

As temperaturas máximas registradas durante o ano foram elevadas, situando-se sempre acima de $30^{\circ}$ centígrados; as temperaturas mínimas foram mais variáveis e apresentaram valores concordantes com os períodos sazonais e com os valores médios mensais, com uma amplitude de variação de $4^{\circ}$ a $20^{\circ}$ centígrados. Os meses mais frios situaram-se entre maio e agosto. Os meses mais secos situaram-se entre julho e agosto, com ausência de chuva e um gradativo aumento do volume pluviométrico de setembro a fevereiro, mês em que foi registrado o maior valor (tab. I).

Foi coletado um total de 1730 indivíduos pertencentes a 26 espécies (tab. II). Os valores estimados para os índices de diversidade entre os córregos foram próximos; também foram próximos os valores dos índices de equitabilidade e as variâncias estimadas (tab. III). Portanto, considerando-se o maior valor de H' (córrego da Água Comprida) e o menor valor de H' (córrego do Buriti), o teste $t$ aplicado a estes índices mostrou não haver diferença significativa $(\mathrm{P}>0,05)$ e os córregos foram tratados em conjunto como ecossistema único.

As espécies consideradas dominantes nos três córregos foram Plagioscion squamosissimus, Pimelodus maculatus, Myleus tiete, Astyanax bimaculatus e A.fasciatus,

Tabela I. Dados climáticos obtidos mensalmente de janeiro de 1997 a fevereiro de 1998, na estação metereológica da Unidade Hidrelétrica de Volta Grande.

\begin{tabular}{|c|c|c|c|c|c|c|c|c|c|c|c|c|c|c|}
\hline Dados climáticos & Jan & Fev & Mar & Abr & Mai & Jun & Jul & Ago & Set & Out & Nov & Dez & Jan & Fev \\
\hline \multicolumn{15}{|l|}{ Temperatura do ar $\left({ }^{\circ} \mathrm{C}\right)$} \\
\hline Máxima & 37,0 & 37,5 & 36,0 & 35,0 & 32,5 & 33,0 & 34,0 & 36,0 & 39,0 & 39,0 & 39,0 & 38,0 & 37,0 & 39,0 \\
\hline Mínima & 19,0 & 17,0 & 18,0 & 13,0 & 9,0 & 4,0 & 9,0 & 8,0 & 11,0 & 15,0 & 20,0 & 19,0 & 13,0 & 20,0 \\
\hline Média & 26,5 & 27,3 & 25,9 & 24,3 & 21,8 & 20,9 & 21,4 & 22,7 & 26,8 & 27,7 & 28,1 & 27,5 & 28,0 & 28,5 \\
\hline \multicolumn{15}{|l|}{ Chuva do } \\
\hline \multicolumn{15}{|l|}{ Pluviômetro (mm) } \\
\hline Média & 15,9 & 4,5 & 6,9 & 0,6 & 1,7 & 3,0 & 0,0 & 0,0 & 1,5 & 3,9 & 9,6 & 8,3 & 7,9 & 11,9 \\
\hline Total & 492,9 & 127,3 & 214,9 & 19,1 & 54,1 & 88,8 & 0,0 & 0,0 & 45,2 & 121,1 & 289,3 & 256,9 & 244,3 & 332,9 \\
\hline
\end{tabular}


Tabela II. Relação das espécies de peixes capturadas nos córregos tributários do reservatório de Volta Grande, rio Grande (MG), em ordem de abundância numérica $(\mathrm{N})$ e os respectivos pesos $(\mathrm{P})$ em quilos.

\begin{tabular}{|c|c|c|c|c|c|c|c|c|}
\hline \multirow[t]{2}{*}{ Espécie } & \multicolumn{2}{|c|}{ Buriti } & \multicolumn{2}{|c|}{ Divisa } & \multicolumn{2}{|c|}{ Água Comprida } & \multicolumn{2}{|c|}{ Total } \\
\hline & $\mathrm{N}$ & $\mathrm{P}$ & $\mathrm{N}$ & $\mathrm{P}$ & $\mathrm{N}$ & $\mathrm{P}$ & $\mathrm{N}$ & $\mathrm{P}$ \\
\hline 1. Plagioscion squamosissimus (Heckel, 1840) & 336 & 44,85 & 110 & 30,90 & 115 & 33,93 & 561 & 109,68 \\
\hline 2. Pimelodus maculatus Lacépède, 1803 & 49 & 14,38 & 235 & 79,41 & 160 & 38,20 & 444 & 131,99 \\
\hline 3. Astyanax bimaculatus (Linnaeus, 1758) & 114 & 3,29 & 50 & 1,47 & 45 & 1,28 & 209 & 6,04 \\
\hline 4. Myleus tiete (Eigenmann \& Norris, 1900) & 34 & 4,06 & 95 & 9,12 & 37 & 4,08 & 166 & 17,26 \\
\hline 5. Cichla cf. ocellaris Bloch \& Schneider, 1801 & 36 & 1,83 & 54 & 5,03 & 5 & 1,51 & 95 & 8,37 \\
\hline 6. Astyanax fasciatus (Cuvier, 1819) & 28 & 0,81 & 30 & 0,83 & 31 & 0,78 & 89 & 2,42 \\
\hline 7. Cyphocharax nagellii (Steindachner, 1882) & 28 & 1,32 & 1 & 0,04 & 1 & 0,04 & 30 & 1,40 \\
\hline 8. Galeocharax knerii (Steindachner, 1879) & 14 & 1,21 & 8 & 0,38 & 4 & 0,21 & 26 & 1,80 \\
\hline 9. Cichla monoculus Spix \& Agassiz, 1831 & 7 & 1,02 & 2 & 1,43 & 8 & 0,64 & 17 & 3,09 \\
\hline 10. Leporinus friderici (Bloch, 1794) & 10 & 7,62 & 2 & 0,47 & 5 & 0,56 & 17 & 8,65 \\
\hline 11. Leporinus octofasciatus (Steindachner, 1917) & 7 & 1,57 & 4 & 0,29 & 6 & 0,48 & 17 & 2,34 \\
\hline 12. Hoplias lacerdae Ribeiro, 1908 & & & 4 & 1,27 & 7 & 1,04 & 11 & 2,31 \\
\hline 13. Hoplias malabaricus (Bloch, 1794) & 2 & 0,46 & 3 & 0,74 & 5 & 1,09 & 10 & 2,29 \\
\hline 14. Schizodon nasutus Kner, 1864 & 4 & 1,50 & 1 & 0,08 & 1 & 0,32 & 6 & 1,90 \\
\hline 15. Astronotus ocellatus Swainson, 1839 & 1 & 0,42 & & & 5 & 1,03 & 6 & 1,45 \\
\hline 16. Leporinus lacustris Campos, 1945 & & & 3 & 0,10 & 3 & 0,16 & 6 & 0,26 \\
\hline 17. Geophagus brasiliensis (Quoy \& Gaimard, 1824) & & & 4 & 0,12 & & & 4 & 0,12 \\
\hline 18. Prochilodus lineatus (Valenciennes, 1847) & & & 3 & 1,25 & & & 3 & 1,25 \\
\hline 19. Gymnotus carapo Linnaeus, 1758 & 2 & 0,15 & 1 & 0,12 & & & 3 & 0,27 \\
\hline 20. Rhynodoras d'orbigny (Kroyer, 1855) & 3 & 0,35 & & & & & 3 & 0,35 \\
\hline 21. Tilapia rendalli (Bougenger, 1897) & & & 2 & 0,66 & 1 & 0,67 & 3 & 1,33 \\
\hline 22. Iheringichthys labrosus (Kroyer, 1874) & & & 2 & 0,27 & & & 2 & 0,27 \\
\hline 23. Leporinus elongatus Valenciennes, 1849 & & & & & 2 & 0,54 & 2 & 0,54 \\
\hline 24. Crenicichla britskii Kullander, 1982 & & & 1 & 0,18 & & & 1 & 0,18 \\
\hline 25. Rhamdia quelen (Quoy \& Gaimard, 1824) & 1 & 0,23 & & & & & 1 & 0,23 \\
\hline 26. Cyphocharax modestus (Fernandes-Yepez, 1948) & 1 & 0,04 & & & & & 1 & 0,04 \\
\hline Total & 677 & 85,11 & 613 & 133,50 & 440 & 85,89 & 1730 & 304,50 \\
\hline
\end{tabular}

Tabela III. Índice de diversidade de Shannon (H'), variância (VAR H') e o índice de equitabilidade (E) para os locais de coleta.

\begin{tabular}{lccc}
\hline Parâmetros & Buriti & Divisa & Água Comprida \\
\hline H' & 0,7653 & 0,8155 & 0,8201 \\
VAR H' & 0,0004 & 0,0004 & 0,0005 \\
E & 0,2648 & 0,2679 & 0,2648 \\
\hline
\end{tabular}

Tabela IV. Freqüência de estádios de maturidade das espécies dominantes nos ribeirões, durante o período de amostragem (1997 e 1998).

\begin{tabular}{lccrc}
\hline & \multicolumn{4}{c}{ Estádio de maturidade } \\
Espécie & $\% \mathrm{~A}$ & $\% \mathrm{~B}$ & $\% \mathrm{C}$ & $\% \mathrm{D}$ \\
\hline Plagioscion squamosissimus & 60,9 & 23,3 & 15,0 & 0,8 \\
Pimelodus maculatus & 46,5 & 50,8 & 2,1 & 0,6 \\
Myleus tiete & 12,3 & 38,6 & 49,1 & 0,0 \\
Astyanax bimaculatus & 8,5 & 53,8 & 36,8 & 0,9 \\
Astyanax fasciatus & 8,1 & 64,9 & 27,0 & 0,0 \\
\hline
\end{tabular}

que também demonstraram poder ter atividade reprodutiva nesses ambientes pela frequiência de fêmeas maduras, exceto P. maculatus (tab. IV).

O período reprodutivo menos intenso ocorreu no inverno (junho de 1997) quando somente 
Tabela V. Frequiência de indivíduos com ovários maduros $\left(\%\right.$ C), valores médios de $\mathrm{RGS}_{\mathrm{c}}$ para fêmeas maduras e da porcentagem da $\mathrm{RGS}_{\text {max }}$ para esses indivíduos, para as espécies dominantes nos ribeirões, por período de coleta.

\begin{tabular}{|c|c|c|c|c|c|c|}
\hline \multirow[t]{2}{*}{ Espécie $\left(\right.$ RGS $\left._{\text {máx }}\right)$} & \multicolumn{3}{|c|}{ Abril/97 } & \multicolumn{3}{|c|}{ Junho/97 } \\
\hline & $\% \mathrm{C}$ & $\mathrm{RGS}_{\mathrm{c}}$ & $\% \mathrm{RGS}_{\max }$ & $\% \mathrm{C}$ & $\mathrm{RGS}_{\mathrm{c}}$ & $\% \mathrm{RGS}_{\max }$ \\
\hline \multicolumn{7}{|l|}{ Pimelodus maculatus $(4,3)$} \\
\hline Myleus tiete $(9,9)$ & 27,3 & 2,9 & 29,3 & 20,0 & 5,8 & 58,6 \\
\hline Astyanax bimaculatus $(13,3)$ & 9,5 & 7,5 & 56,4 & & & \\
\hline \multicolumn{7}{|l|}{ Astyanax fasciatus $(11,9)$} \\
\hline & \multicolumn{3}{|c|}{ Setembro/97 } & \multicolumn{3}{|c|}{ Dezembro/97 } \\
\hline Plagioscion squamosissimus $(3,4)$ & 15,8 & 0,8 & 23,5 & 16,7 & 0,8 & 23,5 \\
\hline Pimelodus maculatus $(4,3)$ & 2,1 & 2,2 & 51,2 & 12,2 & 2,3 & 53,5 \\
\hline Myleus tiete $(9,9)$ & 46,7 & 5,1 & 51,5 & 37,5 & 5,1 & 59,6 \\
\hline Astyanax bimaculatus $(13,3)$ & 40,9 & 6,8 & 51,1 & 81,3 & 5,1 & 38,3 \\
\hline \multirow[t]{2}{*}{ Astyanax fasciatus $(11,9)$} & & & & 34,6 & 9,1 & 76,5 \\
\hline & \multicolumn{3}{|c|}{ Fevereiro/98 } & & & \\
\hline Plagioscion squamosissimus $(3,4)$ & 9,1 & 2,3 & 67,7 & & & \\
\hline \multicolumn{7}{|l|}{ Pimelodus maculatus (4.3) } \\
\hline Myleus tiete $(9,9)$ & 35,5 & 4,8 & 48,5 & & & \\
\hline Astyanax bimaculatus $(13,3)$ & 34,6 & 8,7 & 65,4 & & & \\
\hline Astyanax fasciatus $(11,9)$ & 69,2 & 8,0 & 67,2 & & & \\
\hline
\end{tabular}

Tabela VI. Categorias reprodutivas em que são enquadradas as espécies dominantes nos ribeirões, por período de coleta e o índice de atividade reprodutiva no sistema.

\begin{tabular}{lcccc}
\hline \multicolumn{1}{c}{ Espécie } & Abril/97 & Junho/97 & Setembro/97 & Dezembro/97 \\
\hline Plagioscions squamosissimus & DM & SAR & MI & MI \\
Pimelodus maculatus & SAR & SAR & DO & DO \\
Myleus tiete & MI & DM & DM & DM \\
Astyanax bimaculatus & DO & SAR & DM & DM \\
Astyanax fasciatus & SAR & SAR & SAR & DM \\
DM - desova massiva & $1 \times 4=4$ & $1 \times 4=4$ & $2 \times 4=8$ & $3 \times 4=12$ \\
DO - desova ocasional & $1 \times 2=2$ & $0 \times 2=0$ & $1 \times 2=2$ & $1 \times 2=2$ \\
MI - maturação incipiente & $0 \times 1=0$ & $0 \times 1=0$ & $1 \times 1=1$ & $1 \times 1=1$ \\
SAR - sem atividade reprodutiva & $2 \times 0=0$ & $4 \times 0=0$ & $1 \times 0=0$ & $0 \times 0=0$ \\
IR - índice de atividade reprodutiva & 1,2 & 0,8 & 2,2 & 3,0 \\
\hline
\end{tabular}

Tabela VII. Comprimento médio de primeira maturação em centímetros $\left(\mathrm{L}_{50}\right)$, freqüência das fases jovem e adulta (\% J, \% A), número de indivíduos coletados $(\mathrm{N})$ e número de adultos $(\mathrm{NA})$, para as espécies de peixes dominantes nos ribeirões.

\begin{tabular}{|c|c|c|c|c|c|}
\hline \multirow[b]{2}{*}{ Espécie } & \multirow[b]{2}{*}{$\mathrm{L}_{50}$} & \multicolumn{2}{|c|}{ Fase } & \multicolumn{2}{|c|}{ Estrutura } \\
\hline & & $\% \mathrm{~J}$ & $\% \mathrm{~A}$ & $\mathrm{~N}$ & NA \\
\hline Plagioscion squamosissimus & 26,0 & 58,8 & 41,2 & 131 & 54 \\
\hline Pimelodus maculatus & 30,6 & 46,2 & 53,8 & 331 & 178 \\
\hline Myleus tiete & 15,0 & 31,6 & 68,4 & 79 & 54 \\
\hline Astyanax bimaculatus & 10,5 & 6,0 & 94,0 & 117 & 110 \\
\hline Astyanax fasciatus & 12,0 & 54,1 & 45,9 & 61 & 28 \\
\hline Porcentagem de jovens e adultos & & 41,0 & 59,0 & & \\
\hline
\end{tabular}

\begin{tabular}{lll}
\hline Total & 719 & 424
\end{tabular}

M. tiete teve atividade reprodutiva, estendendo-se esta por um período bastante longo. As demais espécies desenvolveram suas atividades reprodutivas de setembro a abril (tab. V). Adotando-se como valor significativo da intensidade reprodutiva da espécie considerada, aquele 
Tabela VIII. Descrição da situação constatada para a atividade das espécies de peixes dominantes nos córregos, considerando-se a intensidade reprodutiva (IR), valores médios dos graus de repleção (GR) e de gordura acumulada (GA), proporção entre jovens e adultos (\%J : \%A).

\begin{tabular}{llllll}
\hline Espécie & IR & GR & GA & $\% \mathrm{~J}: \% \mathrm{~A}$ & Tipo de utilização \\
\hline Plagioscion squamosissimus & baixa & 1,6 & 1,1 & $\% \mathrm{~J}>\%$ A área de alimentação/crescimento \\
Pimelodus maculatus & ausente & 1,8 & 1,8 & $\% \mathrm{~J}<\%$ A área de alimentação/crescimento \\
Myleus tiete & alta & 2,1 & 2,3 & $\% \mathrm{~J}<\%$ A área de reprodução \\
Astyanax bimaculatus & alta & 1,6 & 1,3 & $\% \mathrm{~J}<\%$ A área de reprodução \\
Astyanax fasciatus & média & 1,5 & 2,0 & $\% \mathrm{~J}>\%$ A área de reprodução e alimentação/ crescimento \\
Médias & alta & 1,7 & 1,7 & $\% \mathrm{~J}<\%$ A área de reprodução \\
\hline
\end{tabular}

com frequiência igual ou superior a $15 \%$ de indivíduos com gônadas maduras (\% C) e do estado funcional desses ovários (\% $\mathrm{RGS}_{\text {máx }}$ ) igual ou superior a $30 \%$ têm-se: P. squamosissimus apresentou desova massiva em abril de 1997; P. maculatus não demonstrou ter atividade reprodutiva intensa nesses ambientes; $M$. tiete teve desova massiva de junho de 1997 a fevereiro de 1998; A. bimaculatus demonstrou ter desova massiva de setembro de 1997 a fevereiro de 1998 e A. fasciatus de dezembro de 1997 a fevereiro de 1998. Considerando-se o índice de intensidade reprodutiva (IR) para as espécies dominantes no ecossistema verificou-se que os períodos mais intensos foram de dezembro a fevereiro (tabs. V, VI).

Analisando-se as espécies dominantes no ecossistema verificou-se predominância de indivíduos adultos (tab. VII). A espécie P. squamosissimus teve uma intensidade reprodutiva baixa e frequiência de jovens superior à de adultos, sendo que a área para essa espécie foi considerada como de alimentação e crescimento. A atividade reprodutiva para P. maculatus foi ausente e a área foi considerada como sendo de alimentação e crescimento. $M$. tiete teve intensidade reprodutiva alta e a freqüência de jovens foi inferior à de adultos, indicando a área de reprodução; por outro lado, foram altos também os graus médios de repleção do estômago e de gordura acumulada na cavidade visceral, sugerindo que a espécie deva utilizar-se desse ecossistema como área de alimentação, além de área de reprodução. As espécies $A$. bimaculatus e A.fasciatus tiveram intensidade reprodutiva alta e frequiência de jovens inferior à de adultos, indicando ser esta uma área de reprodução para essas espécies. Portanto, verificou-se que para o conjunto de espécies dominantes nesse ecossistema seja a área de reprodução para a maioria delas (tab. VIII).

\section{DISCUSSÃO}

A diversidade de espécies foi baixa nas áreas do reservatório de Volta Grande, em relação a outros reservatórios. No reservatório de Itaipu, em três locais de coleta os valores de H' foram 3, 25, 3, 81 e 3, 31 (ITAIPU BinACiOnAL, 1987). As espécies dominantes $P$. squamosissimus e $P$. maculatus também foram as mais abundantes nas pescarias experimentais e artesanais praticadas na área (BRAGA \& GoMIERO, 1997). Levantamentos feitos no reservatório de Volta Grande em 1985 e 1986 detectaram 47 espécies de peixes das quais três foram introduzidas: Astronotus ocellatus Swainson, 1839, Cichla ocellaris Bloch \& Schneider, 1804 e Colossoma macropomum (Cuvier, 1816) (Eletrobrás, 1995). BraGa \& Gomiero (1997) inventariaram 37 espécies de peixes trabalhando na confluência de córregos com a calha do rio e também na própria calha. O menor número de espécies 
encontrado na presente pesquisa deve-se ao fato de não ter sido utilizado na coletas redes com malhas inferiores a 1,5 centímetros entre-nós ou outros aparelhos de pesca que pudessem capturar representantes da ictiofauna de menor porte. No entanto, nas coletas realizadas em 1985 e 1986 não foi amostrada $P$. squamosissimus, que passou a ser dominante no reservatório dez anos depois.

O número de espécies levantadas nos córregos, utilizando-se dos mesmos tipos de redes empregadas por BRAGA \& GomiERo (1997), foi inferior, totalizando 26 espécies. Dessas, somente cinco foram consideradas dominantes, sendo que três utilizaram os córregos como locais de reprodução. Das espécies dominantes, $P$. maculatus, que é uma das mais abundantes no reservatório de Volta Grande, demonstrou ter desova ocasional nesse ecossistema.

A intensidade reprodutiva concentrou-se na primavera e verão e foi mais intensa no verão. BRAGA \& GOMIERO (1997) verificaram que no verão houve um aumento nas capturas de peixes no reservatório de Volta Grande, sendo que a temperatura foi o fator que correlacionou-se com as capturas. Para os peixes da bacia do Paraná, mesmo para as espécies de período reprodutivo longo, que se estende até o inverno, o período mais intenso situou-se na primavera e verão (ELETROBRÁs, 1994). Neste período, as temperaturas são mais elevadas e as chuvas mais intensas, contribuindo para os processos indutores da desova.

Um dos elos mais importantes do ciclo de vida dos peixes e também de sua dinâmica populacional é a reprodução. Os recursos utilizados pelos indivíduos oriundos do período reprodutivo são fundamentais para o sucesso da classe anual e consequentemente da população como um todo (WоOTTON, 1984). É importante destacar que a antiga condição do rio, antes de seu barramento, condicionava as características adaptativas das espécies com relação à reprodução (SUZuKi \& AgostinHo, 1997). O rio Grande é caracteristicamente um rio de planalto, com uma declividade média de $0,8 \mathrm{~m} / \mathrm{km}$, tendendo a atenuar-se em suas porções mais baixas, com declividade de $0,3 \mathrm{~m} / \mathrm{km}$ (AgostinHo \& ZALEWSKI, 1996). As espécies de peixes adaptadas a ambientes reofílicos são as mais afetadas com a transformação súbita do ambiente lótico para lêntico, pois as adaptações e estratégias reprodutivas estavam associadas ao ambiente lótico. Cabe também ressaltar que as condições atuais podem comprometer a abundância das espécies em vários aspectos, como a utilização do espaço físico, a oferta de alimento, a qualidade da água e do substrato.

Uma espécie amplamente distribuída pela bacia do Paraná, de hábitos sedentários e lênticos, é a traíra, Hoplias malabaricus (Bloch, 1794); essa espécie foi beneficiada pelo represamento do rio Iguaçu (Suzuki \& Agostinho, 1997), no entanto, a sua participação foi baixa no ecossistema analisado, ficando em décimo terceiro lugar. A sua participação no total de espécies capturadas na confluência dos córregos com a calha do rio Grande foi a mesma (BRAGA \& GoMIERO, 1997). Não foi verificado atividade reprodutiva para essa espécie nos córregos analisados.

A espécie mais abundante, $P$. squamosissimus, demonstrou ter desova ocasional em fevereiro e desova massiva em abril nos córregos. Porém, analisando-se conjuntamente a intensidade reprodutiva e a proporção entre jovens e adultos (VAZZOLER, 1996) e também os valores médios dos graus de repleção e de gordura acumulada, verificou-se que para essa espécie, na área estudada, houve uma intensidade reprodutiva baixa, sendo considerada de alimentação e crescimento devido a alta freqüência de jovens em relação 
à de adultos e às condições nutricionais (grau de repleção e gordura acumulada).

A segunda espécie mais abundante, P. maculatus, apresentou desovas ocasionais em setembro e dezembro. A análise conjunta da intensidade reprodutiva, proporção entre jovens e adultos e valores médios dos graus de repleção e de gordura acumulada, indicou uma ausência de atividade reprodutiva na área, estando ela relacionada com a alimentação e crescimento. Analisando-se as outras espécies dominantes, A. bimaculatus, A. fasciatus e M. tiete, verificou-se que para estas, a área é de reprodução. Considerandose conjuntamente as espécies dominantes concluiu-se que os ribeirões contribuem como áreas reprodutivas para algumas espécies. No entanto, outras espécies, em geral as mais abundantes, utilizam-se de outras áreas para a reprodução.

Agradecimentos. À Companhia Energética de Minas Gerais (Cemig) e aos funcionários vinculados ao laboratório de reprodução de peixes pelo auxílio prestado durante a execução deste projeto. Ao CNPq e FAPESP pelos auxílios concedidos desde a implantação até o término do projeto.

\section{REFERÊNCIAS BIBLIOGRÁFICAS}

Agostinho, A. A. \& Zalewski, M. 1996. A planície alagável do alto rio Paraná: importância e Preservação. Maringá, Eduem. 100p.

BARBIERI, G. \& GARAVELLO, J.C. 1981. Sobre a dinâmica da reprodução e da nutrição de Leporinus friderici (Bloch, 1794) na represa do Lobo, Brotas - Itirapina, SP (Pisces, Anostomidae). Anais Sem. Reg. Ecol., São Carlos, 2: 347-387.

Braga, F. M. DE S. \& Gomiero, L. M. 1997. Análise da pesca experimental realizada no reservatório de Volta Grande, rio Grande (MG-SP). Bolm Inst. Pesca, São Paulo, 24: 131-138.

CESP. 1993a. Produção pesqueira e composição das capturas em reservatórios sob concessão da CESP nos rios Tietê, Paraná e Grande, no período de 1986 a 1991. CESP, Sér. Prod. Pesq., São Paulo, 1: 1-73.

_. 1993b. A pescada-do-piauí Plagioscion squamosissimus (Heckel, 1840) (Osteichthyes, Perciformes) nos reservatórios da Companhia Energética de São Paulo - CESP. CESP, Sér. Pesq. Des., São Paulo, 84:1-23.

Eletrobrás. 1994. Seminário sobre fauna aquática e o setor elétrico brasileiro, reuniões temáticas preparatórias: caderno 1-Fundamentos. Rio de Janeiro, Comase. $61 \mathrm{p}$.

- 1995. Seminário sobre fauna aquática e o setor elétrico brasileiro, reuniões temáticas preparatórias: caderno 4-Estudos e Levantamentos. Rio de Janeiro, Comase. 104p.

Gennari Filho, O. \& Braga, F. M. DE S. 1996. Fecundidade e desova de Astyanax bimaculatus e A. schubart (Characidae, Tetragonopterinae) na represa de Barra Bonita, rio Piracicaba (SP). Revta UNIMAR, Maringá, 18 (2):241-254.

Godoy, M. P. DE. 1975. Peixes do Brasil - Subordem Characoidei, 3. Piracicaba, Franciscana. 226p.

ItaiPu Binacional. 1987 Ictiofauna e Biologia Pesqueira. Maringá, Imprensa Universitária v. 1. 306p.

MagurRAn, A. E. 1991. Ecological diversity and its measurement. London, Chapman \& Hall. 179p.

Rolla, M. E.; Rosa, S. G. et al. 1990. Composição físico química e biológica do sedimento do reservatório de Volta Grande, Minas Gerais/São Paulo. Acta Limnol. Brasil., São Paulo, 3:201-218.

Santos, G. B. \& Formagio, P. S. 2000. Estrutura da ictiofauna dos reservatórios do rio Grande, com ênfase no estabelecimento de peixes piscívoros. Inf. Agrop., Belo Horizonte, 21(2):98-106.

Suzuki, H. I. \& Agostinho, A. A. 1997. Reprodução de peixes do reservatório de Segredo. In: Agostinho, A. A. \& Gomes, L. C. Reservatório de Segredo, bases ecológicas para o manejo. Maringá. Eduem. p. 163-182.

VAZZOLER, A.E. A. DE M. 1996. Biologia e reprodução de peixes teleósteos: teoria e prática. Maringá. Eduem. 169 p.

Wootton, R. J. 1984. Introduction: strategies and tacties in fish reproduction. In: PotTs, G.W. \& Wooton, R. J.

Fish reproductions: strategies and tacties. London, Academic Press. p. 1-12.

Recebido em 30.07.2000; aceito em 26.03.2001. 